\title{
Asteroseismology of the visual binary 70 Ophiuchi $^{\star}$
}

\author{
F. Carrier and P. Eggenberger
}

Observatoire de Genève, 51 chemin des Maillettes, 1290 Sauverny, Switzerland

e-mail: fabien.carrier@obs.unige.ch

Received 8 November 2005 / Accepted 29 December 2005

\section{ABSTRACT}

Context. Convection in stars excites resonant acoustic waves. The frequencies of these oscillations depend on the sound speed inside the star, which in turn depends on density, temperature, gas motion, and other properties of the stellar interior. Therefore, analysis of the oscillations provides an unrivaled method to probe the internal structure of a star.

Aims. Solar-like oscillations in the primary of the visual binary 70 Ophiuchi are investigated.

Methods. 70 Ophiuchi A was observed with the HARPS spectrograph mounted on the 3.6-m telescope at the ESO La Silla Observatory (Chile) during 6 nights in July 2004 allowing us to collect 1758 radial velocity measurements with a standard deviation of about $1.39 \mathrm{~m} \mathrm{~s}^{-1}$.

Results. The power spectrum of the high precision velocity time series clearly presents several identifiable peaks between 3 and $6 \mathrm{mHz}$ showing regularity with a large spacing of $\Delta v=161.7 \pm 0.3 \mu \mathrm{Hz}$. Fourteen individual modes were identified with amplitudes in the range 11 to $14 \mathrm{~cm} \mathrm{~s}^{-1}$.

Key words. stars: individual: $70 \mathrm{Oph}$ - stars: oscillations - techniques: radial velocities

\section{Introduction}

Analysis of the oscillation spectrum provides an unrivaled method to probe the stellar internal structure, since the frequencies of these oscillations depend on the sound speed inside the star, which in turn depends on density, temperature, gas motion, and other properties of the stellar interior. The prime example of such a probe is the Sun: the five-minute oscillations have provided a wealth of information about the solar interior. The Sun oscillates simultaneously in many modes with periods of about $5 \mathrm{~min}$ and Doppler amplitudes of about $23 \mathrm{~cm} \mathrm{~s}^{-1}$ for the strongest modes. Observation of thousands of oscillation frequencies and comparing them with theoretical calculations has led to significant revisions of solar models.

These results stimulated various attempts to detect a similar signal on other solar-like stars and the stabilized spectrographs have achieved the accuracy needed for solar-like oscillation detection by means of radial velocity measurements (Carrier et al. 2003). However, a major asteroseismological difficulty is the confrontation of observations and theoretical models (Eggenberger et al. 2004b, 2005). The observational measurements available for an isolated star - such as the effective temperature, the metallicity, the luminosity, and eventually the interferometric radius, combined with oscillation frequencies - provide strong constraints to the global parameters of

^ Based on observations collected at the 3.6-m telescope at La Silla Observatory (ESO, Chile: program 073.D-0590). the star but are often not sufficient to unambiguously determine an adequate model and to really test the physics of the models. The additional constraints imposed by the binary nature, namely the same age and initial chemical composition, are extremely valuable for accurately determining the properties of a binary system. Moreover, in the case of binaries, the masses of both components are accurately known by combining visual and spectroscopic orbits. A primary target for the search for p-mode oscillations in such a system is the bright $\mathrm{K} 0$ dwarf 70 Ophiuchi A (HD 165341A), in addition to the $\alpha$ Cen system (Bouchy \& Carrier 2002; Carrier \& Bourban 2003; Bedding et al. 2004; and Kjeldsen et al. 2005) and Procyon (Eggenberger et al. 2004a; Martic et al. 2004).

In this paper, we report Doppler observations of 70 Ophiuchi A made with the HARPS spectrograph resulting in the detection of p-mode oscillations. The observations and data reduction are presented in Sect. 2, the acoustic spectrum analysis and the mode identification in Sect. 3, and the conclusion is given in Sect. 4.

\section{Observations and data reduction}

With a $V$-magnitude of $4.1,70 \mathrm{Oph} \mathrm{A}$ is the main component of a spectroscopic visual binary composed of a $\mathrm{K} 0$ and a K5 dwarf with an orbital period of 88.38 yr (Pourbaix 2000). It was observed over 6 nights in July 2004 with the HARPS spectrograph (Pepe et al. 2002) mounted on the 3.6-m telescope at La Silla 


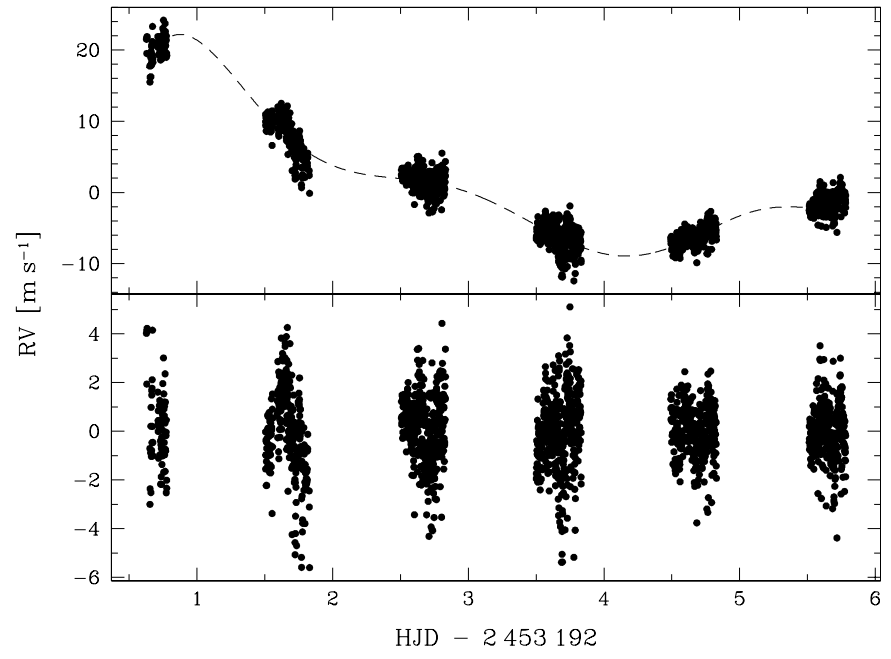

Fig. 1. Radial velocity measurements of 70 Oph A. Top: a constant value of $-5.71 \mathrm{~km} \mathrm{~s}^{-1}$ was removed from the original data. Bottom: an 8 -order polynomial fit was subtracted (dashed line on the top window).

Table 1. Distribution and dispersion of Doppler measurements.

\begin{tabular}{clll}
\hline \hline Date & No. spectra & No. hours & $\sigma\left(\mathrm{m} \mathrm{s}^{-1}\right)$ \\
\hline $2004 / 07 / 05$ & 81 & 3.58 & 1.54 \\
$2004 / 07 / 06$ & 290 & 7.78 & 1.76 \\
$2004 / 07 / 07$ & 350 & 7.80 & 1.39 \\
$2004 / 07 / 08$ & 390 & 7.95 & 1.52 \\
$2004 / 07 / 09$ & 331 & 8.07 & 1.00 \\
$2004 / 07 / 10$ & 316 & 6.45 & 1.11 \\
\hline
\end{tabular}

Observatory (ESO, Chile). We took sequences of 40-60 s exposures, depending on the airmass and the extinction, with a dead time of $30 \mathrm{~s}$ in-between. In total, 1758 spectra were collected with a typical signal-to-noise ratio $(S / N)$ in the range of $120-220$ at $530 \mathrm{~nm}$. During the stellar exposures, the spectrum of a thorium lamp carried by a second fiber was simultaneously recorded in order to monitor the spectrograph's stability. The spectra obtained were extracted on-line. The radial velocities were computed by weighted cross-correlation with a numerical mask constructed from a K5 dwarf spectrum. They were also determined by the optimum-weight procedure (Connes 1985; Carrier et al. 2001) but without significant gain.

The modulation of the Doppler measurements is due to the binarity of the star (see Fig. 1). Thus, an 8-order polynomial fit was subtracted from the raw data to best eliminate the Keplerian movement and variations caused by temperature fluctuations of the instrument during a few days due to a power failure that happened just before our observation run. This high-pass filtering does not affect the results of the pmodes analysis: indeed, a 2 or an 8-order polynomial fit lead to the same power spectrum above $2 \mathrm{mHz}$. The rms scatter of this "new" time series is $1.39 \mathrm{~m} \mathrm{~s}^{-1}$ (see Table 1), the solar-like oscillations representing a non-neglecting part of this number.

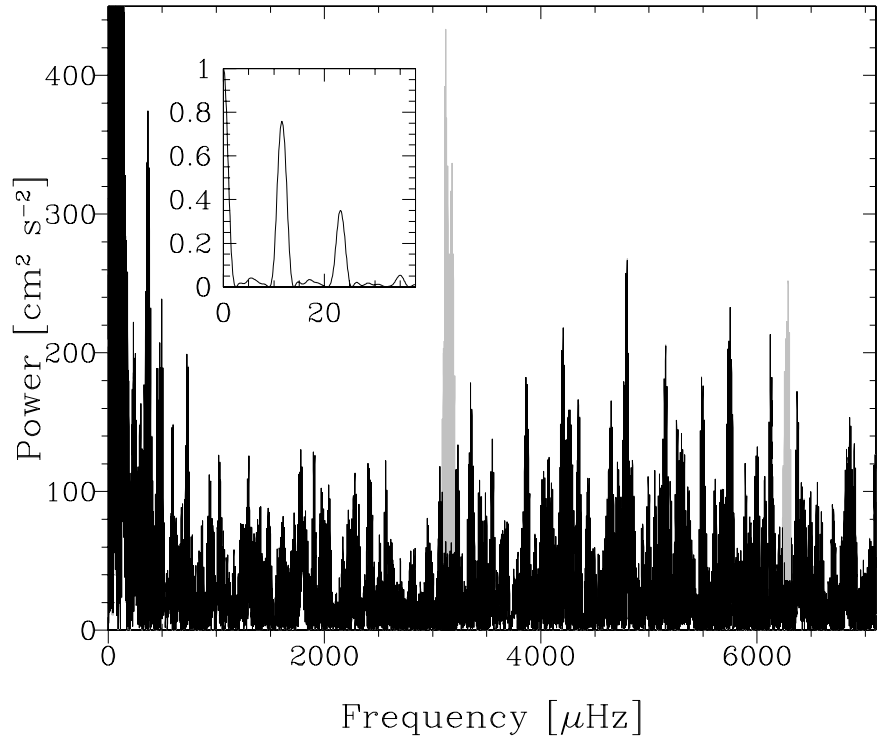

Fig. 2. Power spectrum of 70 Oph A. The high peak in gray (and twice this value) should be due to a technical problem (probably due to a hard point of the telescope). The Nyquist frequency has a value of $7.14 \mathrm{mHz}$. The window function is shown in the inset with daily aliases at 11.57 and $23.15 \mu \mathrm{Hz}$.

\section{Power spectrum analysis}

In order to compute the power spectrum of the velocity time series, we use the Lomb-Scargle modified algorithm (Lomb 1976; Scargle 1982). Its time scale gives a formal resolution of $2.2 \mu \mathrm{Hz}$. The resulting periodogram, shown in Fig. 2, exhibits a series of peaks between 3 and $6 \mathrm{mHz}$, exactly where the solarlike oscillations are expected for this star. Typically for such a power spectrum, the noise has two components:

- At high frequencies it is flat, indicative of the Poisson statistics of photon noise. The mean white noise level $\sigma_{\text {pow }}$ calculated between 1.5 and $2.5 \mathrm{mHz}$ is $0.0026 \mathrm{~m}^{2} \mathrm{~s}^{-2}$, namely $\sigma_{\text {amp }}=\sqrt{\sigma_{\text {pow }} * \pi / 4}=4.5 \mathrm{~cm} \mathrm{~s}^{-1}$ in amplitude (Kjeldsen \& Bedding 1995). With 1758 measurements, this high frequency noise corresponds to $\sigma_{R V}=\sqrt{N \sigma_{\text {pow }} / 4}=$ $1.07 \mathrm{~m} \mathrm{~s}^{-1}$. This radial velocity uncertainty is larger than the uncertainty due to photon noise, which is estimated at $0.25-0.4 \mathrm{~m} \mathrm{~s}^{-1}$. This discrepancy could be explained by the bad seeing and the technical problems occurring during the observation run. However, part of the noise should have a stellar origin (like granulation).

- Towards the lowest frequencies, the power should scale inversely with frequency squared, as expected for instrumental instabilities. Moreover, this star shows low frequencies, not completely removed by the polynomial fit, due to the Keplerian movement.

We note that a high peak near $3.1 \mathrm{mHz}$ and a smaller one nearly twice this value are widely decentered with regard to the others (see Fig. 2). The ESO staff pointed out that a large noise peak could occur with a period of about $6 \mathrm{~min}$ (near $3 \mathrm{mHz}$ and multiple frequencies at 6 and $9 \mathrm{mHz}$ ). This noise is not completely understood at present; it could be due to the guiding, although this seems unlikely given that the guiding correction was 


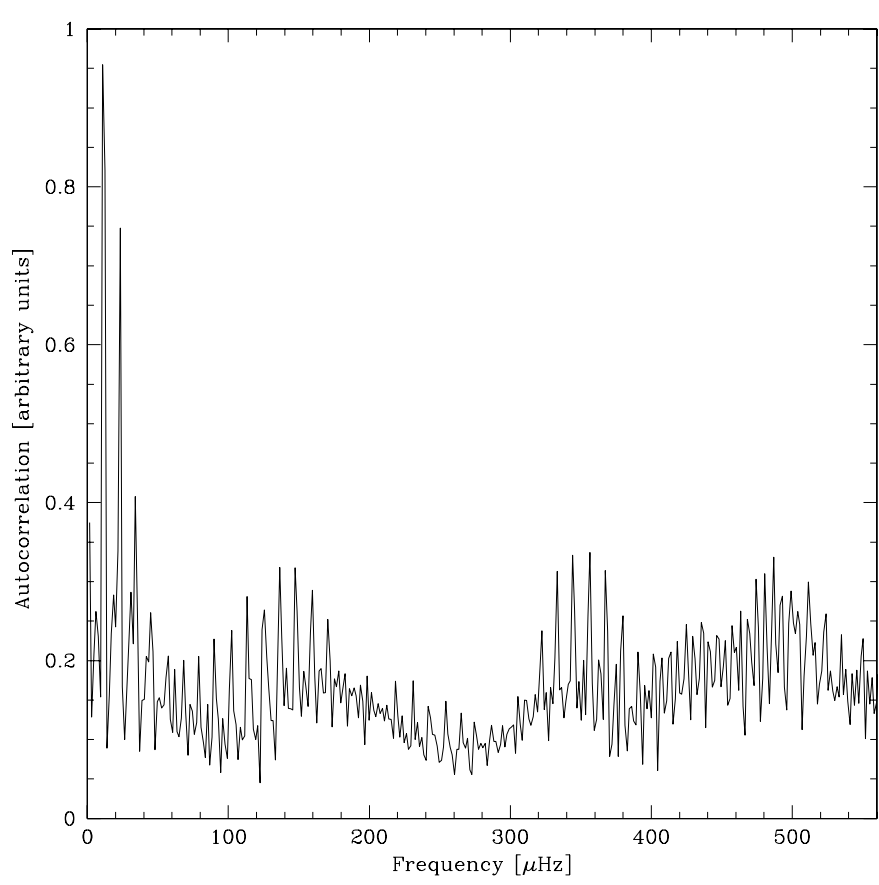

Fig. 3. Autocorrelation of the power spectrum of 70 Oph A with a threshold of $80 \mathrm{~cm}^{2} \mathrm{~s}^{-2}$.

applied every $5 \mathrm{~s}$, or to the telescope. Afterwards, we thus limit our p-modes research between 3.2 and $6 \mathrm{mHz}$.

\subsection{Search for a comb-like pattern}

In solar-like stars, p-mode oscillations of low-degree are expected to produce a characteristic comb-like structure in the power spectrum with mode frequencies $v_{n, \ell}$ reasonably well approximated by the asymptotic relation (Tassoul 1980):

$v_{n, \ell} \approx \Delta v\left(n+\frac{\ell}{2}+\epsilon\right)-\ell(\ell+1) D_{0}$.

Here, $D_{0}$, is sensitive to the sound speed near the core and is equal to $\frac{1}{6} \delta v_{02}$ when the asymptotic relation holds exactly; and $\epsilon$ is sensitive to the surface layers. The quantum numbers $n$ and $\ell$ correspond to the radial order and the angular degree of the modes, and $\Delta v$ and $\delta v_{02}$ to the large and small separations. To search for periodicity in the power spectrum, an autocorrelation with a threshold of $80 \mathrm{~cm}^{2} \mathrm{~s}^{-2}$ (all values below this limit are fixed to zero) is calculated and presented in Fig. 3. Each peak of the autocorrelation corresponds to a structure present in the power spectrum. The three strong peaks at low frequency at about $11.5,23$, and $35 \mu \mathrm{Hz}$ correspond to the daily aliases. The next dominant peaks appear between 120 and $170 \mu \mathrm{Hz}$, and one of them corresponds to the large spacing. Note that the external parameters of $70 \mathrm{Oph} \mathrm{A}$, scaling from the solar case (Kjeldsen \& Bedding 1995), give a large frequency spacing between 150 and $170 \mu \mathrm{Hz}$. Indeed, by refining the orbit of the system and measuring the individual magnitudes and effective temperatures (for both components), we obtain the following parameters for $70 \mathrm{Oph} \mathrm{A}$ : a mass near $0.87 M_{\odot}$, a luminosity of $0.52 L_{\odot}$, an effective temperature of $5300 \mathrm{~K}$, and a solar metallicity. Details about these parameters are postponed to the theoretical paper (Eggenberger et al., in preparation). Other important peaks in the autocorrelation are situated between 320 and $380 \mu \mathrm{Hz}$, corresponding to twice the large separation. The large spacing will then correspond, knowing the domain of one time and twice the large spacing, to half of $333.8 \pm 11.57 \mu \mathrm{Hz}$, namely the two strong peaks at about 161.5 and $172 \mu \mathrm{Hz}$ and the small one at $167 \mu \mathrm{Hz}$. To consolidate our solutions we selected all those peaks between 3.2 and $6 \mathrm{mHz}$ with an amplitude greater than $10 \mathrm{~cm} \mathrm{~s}^{-1}$ and tried to build echelle diagrams with different large separations. Solutions showing well-aligned frequencies are found for large spacings of about 161.5, 167, and $172 \mu \mathrm{Hz}$.

\subsection{Mode identification}

The method used to extract mode frequencies is described well in Carrier et al. (2005a,b). The frequencies are extracted using an iterative algorithm that identifies the highest peak between 3.2 and $6 \mathrm{mHz}$ and subtracts it from the time series. Note that because of the stochastic nature of solar-like oscillations, a timestring of radial velocities cannot be expected to be perfectly reproduced by a sum of sinusoidal terms. Therefore, using an iterative clean algorithm to extract the frequencies can add additional peaks with small amplitudes due to the finite lifetimes of the modes that we do not know. In the case of 70 Oph A, we iterated the process until all peaks with an amplitude higher than $2.5 \sigma$ in the amplitude spectrum were removed. Then, $\sigma$ represents the noise in the amplitude spectrum and has a value of $4.5 \mathrm{~cm} \mathrm{~s}^{-1}$ (see Sect. 3). This threshold ensures that the selected peaks have only a small chance of being due to noise.

Using the extracted frequencies, we drew echelle diagrams with different large spacings. The best echelle diagrams are found for the large spacings of 161.7 and $172.2 \mu \mathrm{Hz}$. The solution with a large spacing of $167 \mu \mathrm{Hz}$, found in the previous section, is ruled out by the echelle diagram; indeed, the distance between the corresponding $\ell=0$ (or 2) and $\ell=1$ modes is not compatible with such a large separation. The results of the automatic extraction are presented in Table 2 for the two different possible large separations with the corresponding identification. Because of the daily alias of $11.57 \mu \mathrm{Hz}$ introduced by the mono-site observations, we cannot know a priori whether the frequency selected by the algorithm is the right one or an alias. We thus considered that the frequencies could be shifted by $\pm 11.57 \mu \mathrm{Hz}$.

To investigate how many peaks are expected to be due to noise, we conducted simulations in which we analyzed noise spectra containing no signal. For this purpose, a velocity time series was built, using the observational time sampling and radial velocities randomly drawn by assuming a Gaussian noise (Monte-Carlo simulations). The amplitude spectrum of this series was then calculated and peaks with amplitude greater than $2.5,3$, and $3.5 \sigma$ were counted; note that a peak and its aliases are only counted once. The whole procedure was repeated 1000 times to ensure the stability of the results. In this way, we find that the number of peaks due to noise with an amplitude larger than $2.5 \sigma$ is $13.0 \pm 4.6$ in the range $3.2-6 \mathrm{mHz}$, 
Table 2. Identification of extracted frequencies. The signs + or - mean that the peaks have to be shifted to a higher or lower value, respectively, by $11.57 \mu \mathrm{Hz}$.

\begin{tabular}{cccc}
\hline \hline $\begin{array}{c}\text { Frequency } \\
{[\mu \mathrm{Hz}]}\end{array}$ & $\begin{array}{c}\text { Mode ID }\left(\Delta v_{1}\right) \\
161.7 \mu \mathrm{Hz}\end{array}$ & $\begin{array}{c}\text { Mode ID }\left(\Delta v_{2}\right) \\
172.2 \mu \mathrm{Hz}\end{array}$ & $S / N$ \\
\hline 3246.2 & noise & $+\ell=1$ & 3.2 \\
3350.9 & noise & $\ell=0$ & 2.8 \\
3378.1 & $\ell=0$ & noise & 2.6 \\
3552.1 & $-\ell=0$ & noise & 2.6 \\
3862.1 & $\ell=0$ & $\ell=0$ & 3.0 \\
4194.4 & $-\ell=0$ & $+\ell=0$ & 3.2 \\
4258.8 & $\ell=1$ & noise & 2.9 \\
4343.7 & $\ell=0$ & noise & 2.8 \\
4650.4 & $+\ell=2$ & $-\ell=1$ & 2.8 \\
4719.6 & noise & $\ell=0$ & 2.5 \\
4797.9 & noise & $+\ell=1$ & 3.6 \\
4993.2 & $\ell=0$ & $-\ell=1$ & 2.5 \\
5054.4 & $+\ell=1$ & $+\ell=0$ & 2.8 \\
5153.7 & $\ell=0$ & $\ell=1$ & 3.1 \\
5255.2 & noise & $-\ell=0$ & 2.9 \\
5328.5 & $-\ell=0$ & $\ell=1$ & 2.6 \\
5487.3 & $-\ell=2$ & $+\ell=1$ & 2.7 \\
5717.8 & $\ell=1$ & noise & 2.7 \\
5763.3 & noise & $\ell=0$ & 3.4 \\
5890.9 & $-\ell=1$ & noise & 2.6 \\
5990.0 & noise & noise & 2.7 \\
\hline \multicolumn{5}{c}{7 noise peaks } & 7 noise peaks \\
\hline & & &
\end{tabular}

for $3 \sigma$ it is $1.9 \pm 1.4$, and for $3.5 \sigma$ the number of peaks due to noise varies between 0 and 3 with a mean value of 0.2 and a standard deviation of 0.4 . These results are in good agreement with the number of identified noise peaks (see Table 2).

The star 70 Oph A is very similar to $\alpha$ Cen B (see Carrier \& Bourban 2003), the same spectral type and similar large spacing, which has a mean small spacing of $10 \mu \mathrm{Hz}$ in the frequency range $3-4.5 \mathrm{mHz}$. Inspecting the results of the mode identification, we note that the value of the small spacing coming from the identification with the large separation of $172.2 \mu \mathrm{Hz}$ is significantly different from $10 \mu \mathrm{Hz}$. No matter what the identification may be $(\ell=0,1$ or 2 for both lines in the echelle diagram), the small separation will be lower than $6.5 \mu \mathrm{Hz}$ in the frequency range $3-4.5 \mathrm{mHz}$. This value of the small spacing, which is lower than expected, suggests that this identification is less reliable than the one with a large separation of $161.7 \mu \mathrm{Hz}$. Although the solution $\Delta v=172.2 \mu \mathrm{Hz}$ cannot be definitely ruled out, we afterwards consider only the one with a large spacing of $161.7 \mu \mathrm{Hz}$.

The echelle diagram based on the extracted frequencies shows only two lines. It is not possible to disentangle $\ell=2$ from $\ell=0$ modes with our time series, mainly due to the poor resolution $(2.2 \mu \mathrm{Hz})$ : daily aliases of $\ell=0$ modes merge with $\ell=2$ modes, and vice versa. This suggests that the small spacing is close to $10 \mu \mathrm{Hz}$. As a result, some extracted $\ell=2$ frequencies could be shifted by $11.57 \mu \mathrm{Hz}$ and be identified as radial modes and inversely.

Due to the ambiguous identification of $\ell=0,2$ modes, another identification can be found replacing all $\ell=0,2$ by

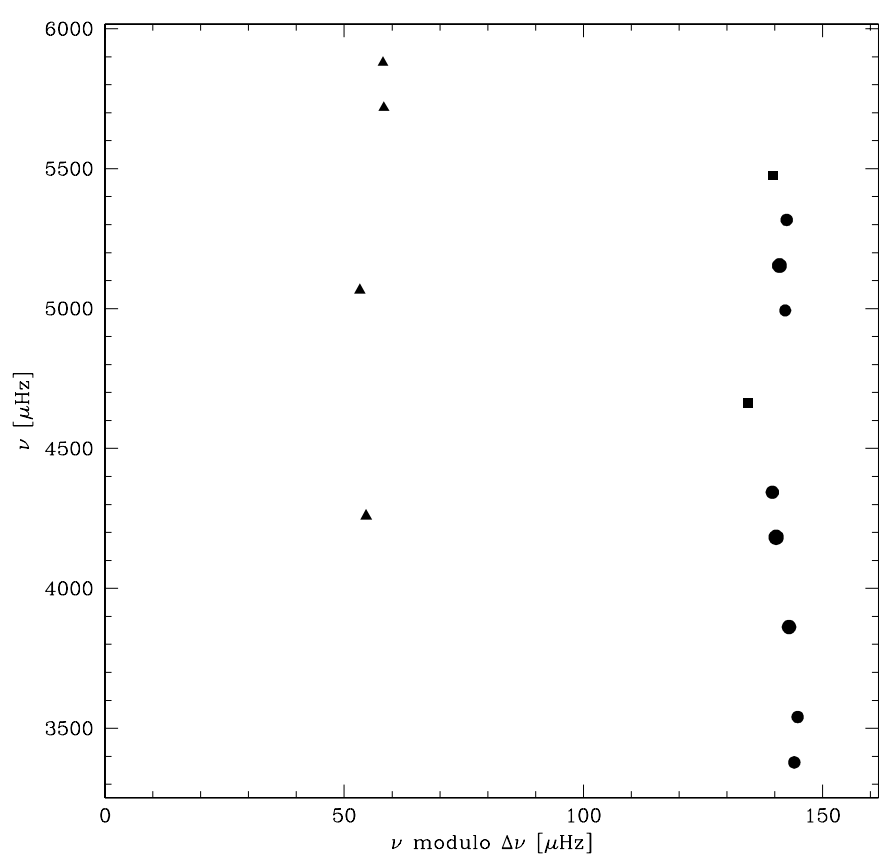

Fig. 4. Echelle diagram of identified modes with a large separation of $161.7 \mu \mathrm{Hz}$. The modes $\ell=2(\boldsymbol{\square}), \ell=0(\bullet)$, and $\ell=1(\boldsymbol{\Delta})$ are represented with a size proportional to their amplitude.

Table 3. Oscillation frequencies (in $\mu \mathrm{Hz}$ ) for the large spacing of $161.7 \mu \mathrm{Hz}$. The frequency resolution of the time series is $2.2 \mu \mathrm{Hz}$, and $n^{\prime}$ is the radial order defined within a constant value. Another possible identification can be done by inverting all $\ell=0,2$ and $\ell=1$ modes, except for the peak at $4662.0 \mu \mathrm{Hz}$, which would be due to noise in this case.

\begin{tabular}{cccc}
\hline \hline$n^{\prime}$ & $\ell=0$ & $\ell=1$ & $\ell=2$ \\
\hline-7 & 3378.1 & & \\
-6 & 3540.5 & & \\
-5 & & & \\
-4 & 3862.1 & & \\
-3 & & & \\
-2 & 4182.8 & 4258.8 & \\
-1 & 4343.7 & & \\
0 & & & \\
1 & & & 4662.0 \\
2 & & & \\
3 & 4993.2 & 5066.0 & \\
4 & 5153.7 & & \\
5 & 5316.9 & & 5 \\
6 & & & 5475.7 \\
7 & & 5717.8 & \\
8 & & 5879.3 & \\
\hline
\end{tabular}

$\ell=1$ modes and all $\ell=1$ by $\ell=2$ modes. In this case, the peak at $4662.0 \mu \mathrm{Hz}$ would be due to noise.

The echelle diagram with the fourteen identified modes is shown in Fig. 4. The frequencies of the modes are given in Table 3. As the curvature of the p-mode alignment could be important above $5.5 \mathrm{mHz}$, both identified high-frequency $\ell=1$ modes can take two different values shifted by $11.57 \mu \mathrm{Hz}$. This should be taken into account when comparing individual frequencies with theoretical models. In the same way, the peak 


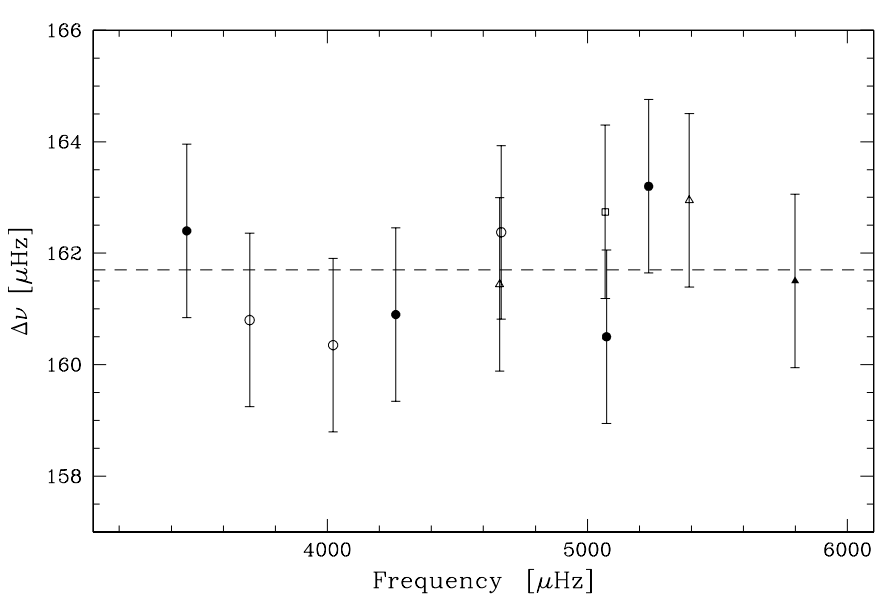

Fig. 5. Large spacing $\Delta v$ versus frequency for p-modes of degree $\ell=0$ $(\bullet)$ and $\ell=1(\boldsymbol{\Lambda})$. Open symbols correspond to large spacing averages taken between non-successive modes. All individual errors are fixed to $\sqrt{2} \times 1.1 \mu \mathrm{Hz}$ (half resolution). The dashed-line corresponds to the mean large spacing of $161.7 \mu \mathrm{Hz}$.

at $5475.7 \mu \mathrm{Hz}$ was identified as a $\ell=2$ mode, taking a probable curvature at high frequency into account, but could just as easily be a radial mode. The large separations are given in Fig. 5 and have a mean value of $161.7 \pm 0.3 \mu \mathrm{Hz}$.

\subsection{Oscillation amplitudes}

Concerning the amplitudes of the modes, theoretical computations predict oscillation amplitudes near $13 \mathrm{~cm} \mathrm{~s}^{-1}$ for a star like 70 Oph A, with mode lifetimes on the order of a few days (Houdek et al. 1999). The amplitudes of the highest peaks are in the range $11-14 \mathrm{~cm} \mathrm{~s}^{-1}$, in agreement with the theoretical value and with the observations of a similar star, $\alpha$ Cen B (Carrier \& Bourban 2003).

\section{Conclusion}

Our observations of 70 Oph A yield a clear detection of p-mode oscillations. Several identifiable modes appear in the power spectrum between 3 and $6 \mathrm{mHz}$ with an average large spacing of $161.7 \mu \mathrm{Hz}$ and a maximal amplitude of $14.5 \mathrm{~cm} \mathrm{~s}^{-1}$. The mono-site observations, coupled to the low resolution of the time series and to the faint signal-to-noise, do not allow us to unambiguously disentangle $\ell=2$ from $\ell=0$ modes. The small spacing seems to be consistent with theoretical predictions for such a star with a value near $10 \mu \mathrm{Hz}$. However, the lack of couples of $\ell=0$ and 2 modes for the same radial order does not give a single identification solution: $\ell=0,2$ can be replaced by $\ell=1$ modes and $\ell=1$ by $\ell=2$ modes. The study of the $70 \mathrm{Oph}$ system, with asteroseismic and non-asteroseismic constraints, has been postponed to a second paper.

Acknowledgements. Part of this work was supported by the Swiss National Science Foundation.

\section{References}

Bedding, T. R., Kjeldsen, H., Butler, R. P., et al. 2004, ApJ, 614, 380 Bouchy, F., \& Carrier, F. 2002, A\&A, 390, 205

Carrier, F., \& Bourban, G. 2003, A\&A, 406, L23

Carrier, F., Bouchy, F., Kienzle, F., et al. 2001, A\&A, 378, 142

Carrier, F., Bouchy, F., \& Eggenberger, P. 2003, Recent Research Developments in Astronomy \& Astrophysics, 1, I, 219, Research Signpost, India

Carrier, F., Eggenberger, P., \& Bouchy, F. 2005a, A\&A, 434, 1085

Carrier, F., Eggenberger, P., D’Alessandro, A., \& Weber, L. 2005b, New Astron., 10, 315

Connes, P. 1985, Ap\&SS, 110, 211

Eggenberger, P., Carrier, F., Bouchy, F., \& Blecha, A. 2004a, A\&A, 422, 247

Eggenberger, P., Charbonnel, C., Talon, S., et al. 2004b, A\&A, 417, 235

Eggenberger, P., Carrier, F., \& Bouchy, F. 2005, New Astron., 10, 195 Houdek, G., Balmforth, N. J., Christensen-Dalsgaard, J., \& Gough, D. O. 1999, A\&A, 351, 582

Kjeldsen, H., \& Bedding, T. R. 1995, A\&A, 293, 87

Kjeldsen, H., Bedding, T. R., Butler, R. P., et al. 2005, ApJ, 635, 1281

Martic, M., Lebrun, J.-C., Appourchaux, T., \& Korzennik, S. G. 2004, A\&A, 418, 295

Lomb, N. R. 1976, Ap\&SS, 39, 447

Scargle, J. D. 1982, ApJ, 263, 835

Pepe, F., Mayor, M., Rupprecht, G., et al. 2002, Messenger, 110, 9

Pourbaix, D. 2000, A\&AS, 145, 215

Tassoul, M. 1980, ApJS, 43, 469 